\title{
Nonlinear excitations in arrays of Bose-Einstein condensates
}

\author{
F.Kh. Abdullaev ${ }^{1 \dagger}$, B.B. Baizakov ${ }^{1 \ddagger}$, S.A. Darmanyan ${ }^{2}$, V.V. Konotop ${ }^{3}$, and M. Salerno ${ }^{4}$ \\ ${ }^{1}$ Physical-Technical Institute, Uzbek Academy of Sciences, 2-b, Mavlyanov str., 700084, Tashkent, Uzbekistan \\ ${ }^{2}$ Institute of Spectroscopy, Russian Academy of Sciences, Moscow reg., Troizk, 142092,Russia \\ ${ }^{3}$ Departmento de Fúsica and Centro de Física da Matéria Condensada, Universidade de Lisboa, \\ Complexo Interdisciplinar, Av. Prof. Gama Pinto 2, Lisboa 1649-003, Portugal \\ ${ }^{4}$ Dipartimento di Fisica "E.R. Caianiello" and Istituto Nazionale di Fisica della Materia (INFM), \\ Universitá di Salerno, via S. Allende, I-84081 Baronissi (SA), Italy
}

(October 26, 2018)

\begin{abstract}
The dynamics of localized excitations in array of Bose-Einstein condensates is investigated in the framework of the nonlinear lattice theory. The existence of temporarily stable ground states displaying an atomic population distributions localized on very few lattice sites (intrinsic localized modes), as well as, of atomic population distributions involving many lattice sites (envelope solitons), is studied both numerically and analytically. The origin and properties of these modes are shown to be inherently connected with the interplay between macroscopic quantum tunnelling and nonlinearity induced self-trapping of atoms in coupled BECs. The phenomenon of Bloch oscillations of these excitations is studied both for zero and non zero backgrounds. We find that in a definite range of parameters, homogeneous distributions can become modulationally unstable. We also show that bright solitons and excitations of shock wave type can exist in BEC arrays even in the case of positive scattering length. Finally, we argue that BEC array with negative scattering length in presence of linear potentials can display collapse.

PACS numbers: 42.65.-k, 42.50. Ar,42.81.Dp
\end{abstract}

\section{INTRODUCTION}

The realization of Bose-Einstein condensates (BEC) and the observation of quantum interference phenomena between two coupled condensates has opened a new fascinating field in physics, with the perspective of getting a better understanding of the complicate behavior of quantum many body systems and with the hope of realizing novel concrete applications of quantum mechanics such as atom interferometers and atom lasers. The existence of periodic localized oscillations in the relative atomic population of one of two coupled condensates (Bloch oscillations), was first predicted in Refs. [1] 3]. The close analogy existing with the Josephson effect was also emphasized, the common origin of these phenomena being the temporal interference of two macroscopic quantum states, leading to current oscillations in Josephson junctions [4], and to oscillations of the atomic population in coupled BECs [5].

Although the generalization of these results to the case of three coupled BECs was considered [6], few theoretical investigations exist on interference phenomena in arrays of coupled BEC's [7]. In a recent paper [8], the problem of Bloch oscillations of bright solitons was investigated in terms of a tight-binding model for BEC arrays with positive scattering length. It is a fortunate situation that the equations arising in this case, formally coincide with those studied in the theory of nonlinear lattices [9]. One can indeed transpose the knowledge gained in these fields, to the field of BEC arrays. Thus, for example, one can expect that the types of elementary excitations which can arise in BEC arrays, as well as their dynamical behavior, strongly depend on the ratio between the coupling of adjacent condensates and the nonlinearity induced by the inter atomic interactions. Keeping the same terminology used in nonlinear lattices theory, one can say that for weak coupling and strong nonlinearity, intrinsic localized modes (ILM), i.e. matter excitations localized on few lattice sites, should exist. These modes could arise in experiments like the ones reported in Ref. [10] in which millions of atoms were trapped in an almost one-dimensional 1D geometry ("cigar" shape geometry), with a small tunnelling term between condensates.

On the other hand, when the coupling constant is comparable with the self-trapping interactions, small amplitude excitations of large size (in comparison with the lattice constant), should arise. These excitations can be identified as lattice envelope solitons (ES) and could be observable in macroscopic quantum interference experiments like the ones reported in Ref. 11] in which, vertical arrays of "pancake"-like BECs, each containing thousands of ${ }^{87} \mathrm{Rb}$ atoms, were coupled through the gravitational field. In this case the interference phenomenon shows up as Bloch oscillations of the ES describing the atomic population along the array (ES dynamical localization). Bloch oscillations of ES type are also possible in horizontal optical lattices induced by two counterpropagating laser beams with a frequency detuning varying linearly in time 12] (this produces a linear potential on the BEC similar to the gravitational field of the vertical traps). In the following we shall call both types of excitations (i.e. ILM or ES) as discrete matter solitons (DMS) whenever it will be clear from the context to which type we refer [13].

The aim of this paper is twofold. From one side, we wish to make a bridge from the theory of nonlinear lat- 
tices to the field of BEC arrays. This will allow to get a better understanding of the types of excitations which can arise in 1D coupled BECs as the physical parameters are varied. From the other side, we wish to expand both theoretically and numerically, on the phenomenon of Bloch oscillations in the case of 1D BEC arrays in presence of uniform backgrounds (with the term "background" here we mean the presence of a uniform distribution of atomic population along the array). In particular, we discuss:

i) the existence of ILM in 1D BEC arrays with positive scattering length;

ii) the existence of ES in BEC arrays with positive scattering lengths;

iii) Bloch oscillations of soliton-like excitations on top of uniform backgrounds in periodic BECs with positive scattering lengths;

iv) the dynamics of BEC arrays with negative scattering length on zero backgrounds.

As to point i) we remark that in contrast with the solitons and breathers discussed of Ref. [8] which are extended on many lattice sites [14], the bright ILM discussed here are highly discrete, i.e. localized on few lattice sites. Since ILM have not been observed in real BEC arrays, this is an implicit call for experimental work to be done in this direction (for experimental evidence of ILM existence in other physical contexts see [15]). Point ii) generalizes the results of [8] to the case of non-zero backgrounds, while in iii) we show that modulational instability can arise as the slope of the linear potential (gravitational field for vertical arrays) is increased. Moreover, we find that for certain parameter values, shock wave excitations can develop. In point vi) we discuss Bloch oscillations of bright solitons both analytically and numerically. All these results will have a close analogy with those derived in the theory of nonlinear lattices on intrinsic localized modes [16 19], envelope solitons 20 22], shock waves [21,23], and Bloch oscillations [24,25].

The paper is organized as follows. In Section II the model of a 1D array of BEC is derived. In Section III we discuss the existence of intrinsic localized BEC solitons, both of even and odd symmetry. In Section IV we use a multi-scale expansion to discuss the stability and dynamics of small amplitude excitations of BEC arrays with positive scattering length against a uniform background and in presence of a linear potential modelling the gravitational field. The linear stability analysis of the background for BEC arrays is investigated, and the region in parameter space where modulational instabilities develop is provided. We show that for particular parameters, shock wave propagation can also develop. In section $\mathrm{V}$ the main characteristic of Bloch oscillations for arrays with negative scattering length, are discussed. Finally, in the last section VI the main results of the paper are summarized.

\section{THE MODEL}

As is well known, in the mean field approximation the wave function of a BEC in a trap potential $V(\mathbf{r})$ satisfies the time-dependent Gross-Pitaevskii equation (GPE)

$i \hbar \frac{\partial \Psi(\mathbf{r}, t)}{\partial t}=\left[-\frac{\hbar^{2}}{2 m} \Delta+V(\mathbf{r})+g_{0}|\Psi(\mathbf{r}, t)|^{2}\right] \Psi(\mathbf{r}, t)$,

where $g_{0}=4 \pi \hbar^{2} a_{s} / m, m$ is the atomic mass, $a_{s}$ is the $s$-wave scattering length which can be either positive (the case of ${ }^{87} \mathrm{Rb}$ atoms, with repulsive interactions, and with $a_{s}=5.5 \mathrm{~nm}$ ) or negative (the case of ${ }^{7} \mathrm{Li}$ atoms, with attractive interactions and $a_{s}=-1.45 \mathrm{~nm}$ ). The trap potential is assumed to be periodic along the $z$-direction

$$
V(\mathbf{r})=V(\mathbf{r}+\lambda \hat{k}),
$$

where $\lambda$ is the spatial period of the potential and $\hat{k}$ is a unitary vector in the $z$-direction. A typical model potential is

$$
V(\mathbf{r})=m g z+V_{0}(x, y) \sin ^{2}(k z)
$$

with experimental parameters $\lambda=2 \pi / k \sim 850 \mathrm{~nm}, V_{0} \sim$ $2.1 \hbar^{2} k^{2} / 2 m$, and with an atomic population in each wells of $N_{0} \approx 10^{3}$ atoms [11].

To make analytical studies it is convenient to look for solutions of Eq. (1) of the form

$$
\Psi(\mathbf{r}, t)=\sum_{n=-\mathcal{N} / 2}^{\mathcal{N} / 2} \psi_{n}(t) \Phi_{n}(\mathbf{r}),
$$

where the summation is performed over the number, $\mathcal{N}$, of minima of the trap potential which is assumed to be even. The functions $\Phi_{n}(\mathbf{r}) \equiv \Phi\left(\mathbf{r}-\mathbf{r}_{n}\right)$ are assumed to be strongly localized around the site $n$ of the potential and normalized to the mean number of atoms in the n-th well $N_{0}=N / \mathcal{N}_{w}$,

$$
\int \bar{\Phi}_{n}(\mathbf{r}) \Phi_{n}(\mathbf{r}) d \mathbf{r}=N_{o}
$$

where $N$ is the total number of atoms and $\mathcal{N}_{w}=\mathcal{N}+1$ the total number of potential wells (hereafter the overbar will denote complex conjugation). This implies that the hopping integral

$$
\left|J_{n, n+1}\right|=\left|\int \bar{\Phi}_{n}(\mathbf{r}) \Phi_{n+1}(\mathbf{r}) d \mathbf{r}\right|=\ll N,
$$

can be neglected, i.e. we assume $J_{n, n+1} \approx 0$ for all $n$. This is analogous to the well known tight binding approximation of solid state physics [26], valid for weakly overlapped condensates. Note that from (5) and (6) the following normalization condition for the functions $\psi_{n}(t)$ is obtained 


$$
\sum_{n=-\mathcal{N} / 2}^{\mathcal{N} / 2}\left|\psi_{n}(t)\right|^{2}=1 .
$$

Substituting (伍) into (伍), multiplying by $\bar{\Phi}_{n}(\mathbf{r})$ and integrating over $\mathbf{r}$, we readily obtain the system of coupled equations

$$
\begin{aligned}
i \hbar \dot{\psi}_{n}= & E_{n} \psi_{n}+U_{n}\left|\psi_{n}\right|^{2} \psi_{n}- \\
& K_{n, n-1} \psi_{n-1}-K_{n, n+1} \psi_{n+1}+\gamma_{0} n \psi_{n},
\end{aligned}
$$

where the overdot means time derivative, and $\gamma_{0}=m g a$ with $a=\lambda / 2$ the distance between adjacent wells. In Eq. (8) we have denoted with

$$
E_{n}=\int\left[\frac{\hbar^{2}}{2 m}\left|\nabla \Phi_{n}\right|^{2}+\left|\Phi_{n}\right|^{2} V(\mathbf{r})\right] d \mathbf{r}
$$

the zero-point energy for the well $n$, with

$$
U_{n}=\frac{g_{0}}{N} \int\left|\Phi_{n}\right|^{4} d \mathbf{r}
$$

the mean-field self-interaction energy, and with

$$
K_{n, n \pm 1}=-\int\left[\frac{\hbar^{2}}{2 m}\left(\nabla \Phi_{n} \nabla \Phi_{n \pm 1}\right)+\left(\Phi_{n} V \Phi_{n \pm 1}\right)\right] d \mathbf{r}
$$

the sum of the kinetic energy and the off-diagonal matrix element of the trap potentials between sites $n$ and $n+1$ (i.e. the coupling constant between neighboring BECs). In the following we shall consider the case of equal constants $E_{n}=E, U_{n}=U, K_{n, n \pm 1}=K$. Introducing the new variables

$$
t \rightarrow \frac{K}{\hbar} t, \quad \psi_{n} \rightarrow\left(\frac{2|U|}{K}\right)^{1 / 2} \overline{\psi_{n}} e^{i\left(\frac{E}{\hbar K}-2\right) t} .
$$

we can rewrite Eq. (8) in dimensionless form as

$$
i \dot{\psi}_{n}+\psi_{n+1}+\psi_{n-1}-2 \psi_{n}+2 \sigma\left|\psi_{n}\right|^{2} \psi_{n}+\gamma n \psi_{n}=0,
$$

with $\sigma=-\operatorname{sign}\left(a_{s}\right)$ and $\gamma=\gamma_{0} / K$. Equation (13) coincides with the well known discrete nonlinear Schrödinger equation [9], extensively investigated in the theory of nonlinear lattices. In the present context, however, because of the normalization condition (7), the boundary conditions for Eq. (13) cannot be choosen arbitrarily. In this paper we consider either periodic boundary condition

$$
\psi(n)=\psi(n+\mathcal{N}+1)
$$

in the case of finite lattices, or zero

$$
\lim _{|n| \rightarrow \infty} \psi(n)=0
$$

or "finite density"

$$
\lim _{n \rightarrow \pm \infty} \psi(n)=\rho e^{ \pm \vartheta}
$$

boundary conditions, in the case of infinite lattices (here $\rho$ and $\vartheta$ are arbitrary constants). Eq. (13) will then have also another integral of motion of the form

$$
H=\sum_{n} \bar{\psi}_{n} \psi_{n-1}-\left|\psi_{n}\right|^{4} .
$$

The mean field tight-binding model in Eq. (13) will be used in the following to study the dynamical properties of both ILM and ES excitations in 1D BEC arrays.

\section{EXISTENCE OF INTRINSIC LOCALIZED MODES IN BEC ARRAYS}

As is well known, in the case of continuous 1D BECs, soliton ground state solutions can exist. These were theoretically predicted in [27] and experimentally observed in the case of positive scattering length (dark solitons) [28]. Bright solitons can exist in BECs only when the effects of focusing nonlinearity balance the ones of effective dispersion, this being possible for attractive inter-particle interactions or negative scattering lengths, only. Since bright solitons are very important for developing BEC applications 29], it is of interest to investigate their existence also in BEC arrays. In this case, as we will see in the following, new possibilities can arise. In Ref. [8] bright solitons of the ES type were numerically investigated in 1D BEC arrays with positive scattering lengths. Here we shall consider the case of bright solitons of ILM type in BEC arrays with both positive and negative scattering lengths. We fix $\gamma=0, \sigma=1$, in Eq. (13), i.e. we consider BECs in horizontal traps with negative scattering lengths. ILM are then expected to exist when the coupling constant (which is responsible in the linear case of the spreading of the BEC wavefunction) is small in comparison with the nonlinear self-trapping interaction. From the normalization condition (5), and from definitions (10), (11), one can estimate $|U| / K=O(N)$, i.e. a stronger nonlinearity corresponds to a larger number of particles. For the sake of analytical developments is convenient to introduce the small parameter

$$
\kappa=\sqrt{\frac{K}{|U|}}=O\left(N^{-1 / 2}\right) \ll 1
$$

representing the ratio between tunnel coupling and nonlinearity, and recast Eq. (13) it in the form:

$$
i \dot{\varphi}_{n}+\kappa\left(\varphi_{n+1}+\varphi_{n-1}\right)+\left|\varphi_{n}\right|^{2} \varphi_{n}=0,
$$

with $\varphi_{n}=\sqrt{\kappa} \psi_{n} e^{2 i t}$ (the overdot here denotes the derivative with respect to $\tau=t / \kappa)$.

In the following we discus ILM solutions of Eq. (19) with different symmetry properties. 


\section{A. Symmetric ILM centered on a site}

Symmetric ILM solutions centered on a site (say $n=0$ )

$$
\varphi_{-n}(\tau)=\varphi_{n}(\tau), \quad\left|\varphi_{0}(\tau)\right|=1,
$$

can be searched in the form

$$
\varphi_{n}=e^{i \omega \tau} \sum_{j=n}^{\infty} \varphi_{n j} \kappa^{j}, \quad \omega=\sum_{j=0}^{\infty} \omega_{j} \kappa^{j},
$$

with the frequency of the local oscillation $\omega$ equal for all sites. Substituting this expansion into Eq. (19) and performing straightforward algebra, we obtain

$$
\omega=1+2 \kappa^{2}-2 \kappa^{6}+2 \kappa^{8}+O\left(\kappa^{10}\right),
$$

with the site amplitude $\varphi_{n}$ satisfying the lattice equations

$$
\begin{aligned}
& \varphi_{1}=\left[\kappa-\kappa^{5}+\kappa^{7}+O\left(\kappa^{9}\right)\right] e^{i \omega \tau}, \\
& \varphi_{2}=\left[\kappa^{2}-\kappa^{4}+\kappa^{8}+O\left(\kappa^{10}\right)\right] e^{i \omega \tau}, \\
& \varphi_{3}=\left[\kappa^{3}-2 \kappa^{5}+\kappa^{7}+O\left(\kappa^{9}\right)\right] e^{i \omega \tau}, \\
& \varphi_{4}=\left[\kappa^{4}-3 \kappa^{6}+O\left(\kappa^{8}\right)\right] e^{i \omega \tau}, \\
& \varphi_{5}=\left[\kappa^{5}+O\left(\kappa^{7}\right)\right] e^{i \omega \tau},
\end{aligned}
$$

from which we see that $\left|\varphi_{n}\right| \gg\left|\varphi_{n+1}\right|$ provided $\kappa \ll 1$.

It is remarkable that the decay of the amplitude $\varphi_{n}$ is exponential as one move away from the $n=0$ site, and in the leading order can be approximated by

$$
\varphi_{n} \approx \exp (i \omega \tau-\eta|n|)
$$

with $\eta$ given by $\exp (-\eta)=\kappa<<1$. Note that in accordance with our scaling, the limit $\kappa \rightarrow 0$ can be viewed as equivalent to the strongly nonlinear limit $N \rightarrow \infty$ of the original physical system. Moreover, from Eq. (23) it follows that a bright onsite centered ILM can have for $\kappa=0.1$ about $91 \%$ of all the atoms concentrated on its central site. For negative scattering lengths this implies that ILM can be stable only if $N<N_{c r}$, where $N_{c r}$ is the critical threshold at which collapse phenomena occur (the effective coupling constant cannot be made smaller than a critical value, this being a feature of BEC arrays with respect to other nonlinear lattices).

To check these results we have performed numerical integrations of Eq. (19) using as initial condition Eq. (23) with $\tau=0$. We used a DOPRI8 integration routine [30], based on a Runge-Kutta scheme of 7th-8th order with automatic stepsize control, so to combine speed with high precision (the relative errors was from $10^{-7}$ up to $\left.10^{-13}\right)$. The integration domain was taken large enough (800 sites) to avoid the influence of boundary conditions. The results are presented in Figs.1 $a, b$ for the case, respectively, of weak and strong coupling among neighboring BECs in the array. We see that in the case of weak coupling the initial atomic population remains stable for arbitrary long times (Fig. 1a), while for strong couplings, the initial distribution of atoms spreads out over the whole system (Fig. 1b).

FIG. 1. Distribution of the normalized atomic population according to numerical solution of Eq. (19) for the case of weak coupling, $\kappa=0.1$, (a) and strong coupling, $\kappa=0.8$ (b): this distribution corresponds to $\tau=10$. The dashed line shows the ILM envelope given by Eq. (23).

\section{B. Anti-symmetric ILM centered on a site}

ILM of anti-symmetric type

$$
\varphi_{0}=0, \quad \varphi_{1}=1, \quad \varphi_{-n}=-\varphi_{n},
$$

can be searched in the form $(n>1)$

$$
\varphi_{n}=e^{i \omega=\tau} \sum_{j=n}^{\infty} \varphi_{n j} \kappa^{j-1}, \quad \omega=\sum_{j=0}^{\infty} \omega_{j} \kappa^{j} .
$$

Direct substitution into Eq. (19) gives

$$
\begin{aligned}
& \omega=1+\kappa^{2}+\kappa^{4}+2 \kappa^{6}+6 \kappa^{8}+O\left(\kappa^{9}\right), \\
& \varphi_{2}=\left[\kappa+\kappa^{3}+2 \kappa^{5}+6 \kappa^{7}+O\left(\kappa^{9}\right)\right] e^{i \omega \tau}, \\
& \varphi_{3}=\left[\kappa^{2}+\kappa^{4}+2 \kappa^{6}+5 \kappa^{8}+O\left(\kappa^{10}\right)\right] e^{i \omega \tau}, \\
& \varphi_{4}=\left[\kappa^{3}+\kappa^{5}+\kappa^{7}+O\left(\kappa^{9}\right)\right] e^{i \omega \tau}, \\
& \varphi_{5}=\left[\kappa^{4}+\kappa^{6}+O\left(\kappa^{8}\right)\right] e^{i \omega \tau}
\end{aligned}
$$

In analogy with the symmetric case, one can ensure that to leading order the shape of the ILM is well described by the function

$$
\varphi_{n} \approx \exp (i \omega \tau-\eta|n-1|), \quad n \geq 1
$$

Direct numerical simulations of Eq. (23) with initial conditions given by Eq. (27)), showed a behavior of the atomic population distribution as a function of the coupling constant, similar to the one reported in Fig. 11.

Other types of bright symmetric and anti-symmetric ILMs, such as the ones which are centered between two sites, can also be constructed. A more detailed analysis of these excitations shows that the stability of ILMs depends on their symmetry property, as well as, on their localization extension 17,19,31. Thus, symmetric onsite centered ILMs are stable but symmetric centered between sites ILMs are unstable, while antisymmetric centered between sites ILM are stable if $\kappa<\kappa_{c r} \approx 0.12$, and unstable if $\kappa>\kappa_{c r}$.

For positive scattering lengths, different families of ILMs (as well as discrete fronts) representing dark ILM 
(i.e. a constant atomic population along the array except for few sites in which the population is decreased), can also be found 17,18,22.

It is important to note that, due to the invariance of Eq. (19) under the transformation $\varphi_{n} \rightarrow(-1)^{n} \varphi_{n}$, $\kappa \rightarrow-\kappa$, we have that the above bright (dark) ILMs, multiplied by a factor $(-1)^{n}$, are also solutions of Eq. (19) for $a_{s}>0\left(a_{s}<0\right)$. This shows Eq. (19) possesses both bright and dark ILM solutions, for any sign of $a_{s}$.

\section{BEC ARRAYS WITH POSITIVE SCATTERING LENGTHS}

From the experiment in Ref. [11] on vertical BEC arrays and from numerical simulations, it is known that the phenomenon of Bloch oscillations of ES can occur. In this section we shall use methods of nonlinear lattices theory 24, 25, 34, 35 to investigate Bloch oscillations of $\mathrm{ES}$ in BEC arrays with positive scattering lengths. For bright ES on zero backgrounds this was done in Ref. [8], so we concentrate here only on the case of ES on top of non zero backgrounds. To this end it is convenient to introduce the background amplitude $\rho$ directly into Eq. (13) with $\sigma=-1$, this giving

$$
\begin{aligned}
& i \dot{\varphi}_{n}+\varphi_{n+1}+\varphi_{n-1}-2 \varphi_{n}+ \\
& 2\left(\rho^{2}-\left|\varphi_{n}\right|^{2}\right) \varphi_{n}+\gamma n \varphi_{n}=0
\end{aligned}
$$

where $\varphi=\psi_{n} \exp \left(-2 i \rho^{2} t\right)$. Let us consider the case of infinite number of lattice sites for which the integrals of motion in Eq. 28) are written as

$$
N_{0}=\sum_{n}\left(\left|\varphi_{n}\right|^{2}-\rho^{2}\right)
$$

and

$$
H=\sum_{n}\left(\left(\bar{\varphi}_{n} \varphi_{n-1}-\rho^{2}\right)-\left(\left|\varphi_{n}\right|^{2}-\rho^{2}\right)^{2}\right) .
$$

Moreover, since the problem has nonzero boundary conditions we must have, for consistency with Eq. (28] [35], that

$$
\varphi_{n} \rightarrow \rho \exp \left[i \Phi_{n}(t)\right] \quad \text { at } \quad n \rightarrow \pm \infty
$$

with the phase $\Phi_{n}(t)$ defined as

$$
\begin{aligned}
\Phi_{n}(t) & =n t \gamma+\chi(t), \\
\chi(t) & =2 t\left(\frac{\sin \gamma t}{\gamma t}-1\right) .
\end{aligned}
$$

The following gauge transformation 34, 35

$$
q_{n}=\varphi_{n} e^{-i \gamma n t},
$$

allows to rewrite Eq. (28) as

$$
i \dot{q}_{n}+e^{i \gamma t} q_{n+1}+e^{-i \gamma t} q_{n-1}-2 q_{n}+2\left(\rho^{2}-\left|q_{n}\right|^{2}\right) q_{n}=0
$$

with boundary conditions

$$
q_{n} \rightarrow \rho \exp [i \chi(t)], \quad \text { at } \quad n \rightarrow \pm \infty .
$$

In the next subsection we use this equation to study the stability of the background of a BEC array in presence of linear potentials $(\gamma \neq 0)$.

\section{A. Linear stability analysis of the background}

Let us consider a solution of Eq. (35) of the form

$$
q_{n}=\left[\rho+\alpha_{n}(t)\right] \exp [i \chi(t)]
$$

where $\left|\alpha_{n}(t)\right| \ll \rho$. Linearizing Eq. (35) around $\rho \exp [i \chi(t)]$ and expanding in Fourier modes

$$
\begin{aligned}
\beta(k, t) & =\sum_{n=-\infty}^{\infty} \alpha_{n}(t) e^{i k n}, \\
\alpha_{n}(t) & =\frac{1}{2 \pi} \int_{0}^{2 \pi} \beta(k, t) e^{-i k n} d k
\end{aligned}
$$

we obtain

$$
\frac{d}{d t}|k, t\rangle=\mathbf{T}_{k}(t)|k, t\rangle
$$

where $\mathbf{T}_{k}(t)$ is a $4 \times 4$ matrix whose nonzero elements are

$$
\begin{aligned}
& T_{k}^{(12)}=-T_{k}^{(21)}=2\left[\cos (\gamma t-k)-\cos (\gamma t)-\rho^{2}\right], \\
& T_{k}^{(34)}=-T_{k}^{(43)}=2\left[\cos (\gamma t+k)-\cos (\gamma t)-\rho^{2}\right], \\
& T_{k}^{(14)}=T_{k}^{(23)}=T_{k}^{(32)}=T_{k}^{(41)}=2 \rho^{2},
\end{aligned}
$$

and with $|k, t\rangle$ a Bloch-Floquet state written in vector form

$$
|k, t\rangle=\left(\begin{array}{c}
\beta_{1}(k, t) \\
\beta_{2}(k, t) \\
\beta_{1}(-k, t) \\
\beta_{2}(-k, t)
\end{array}\right)
$$

(here $\beta(k, t)=\beta_{1}(k, t)+i \beta_{2}(k, t)$ with $\beta_{j}(k, t)$ real).

FIG. 2. Floquet stability analysis of a k-modulated background as a function of $\gamma$. Regions of stability are designated with "+". 
Since $\mathbf{T}_{k}$ is periodic we can use Floquet theory [36] to study the stability. To this end we introduce the matrix $\mathbf{S}_{k}(t)$ as a solution of the equation

$$
d \mathbf{S}_{k} / d t=\mathbf{T}_{k} \mathbf{S}_{k}
$$

satisfying the initial condition $\mathbf{S}_{k}(0)=\mathbf{I}$ (here $\mathbf{I}$ is the $4 \times 4$ unit matrix). It then follows that the background is unstable if $\left|\lambda_{j}(k)\right|>1$, where $\lambda_{j}(k)$ is the eigenvalue of $\mathbf{S}_{k}(2 \pi / \gamma)$ (note that $\mathbf{S}_{k}(t)$ is unimodular, $\operatorname{det} \mathbf{S}_{k}(t) \equiv 1$, so that $\left.\lambda_{1} \lambda_{2} \lambda_{3} \lambda_{4}=1\right)$. In Fig. 2 the results of Floquet analysis, as obtained from numerical integrations of Eq. (40), are reported (in the numerical scheme we used a DOPRI8 procedure to integrate Eq. (40) and computed the spectrum of the matrix $\mathbf{S}_{k}$ by reducing it to Hessenberg form [37]). The stability of the background was also investigated by direct numerical integrations of Eq. (35) taking as initial conditions a uniform background of amplitude $\rho=1$ modulated by a sine wave of wavenumber $k_{n}=2 \pi n / L$ (we used $L=400$ lattice sites).

FIG. 3. Modulational stability of the background at $\gamma=0$, for an initial modulation with $k \sim 0.31$

FIG. 4. Modulational instability at $\gamma=0.5$, for an initial modulation with $k \sim 0.31$

These results are reported in Figs. 3, 1 for the cases $\gamma=0$, and $\gamma=0.5$, respectively. We see that in presence of the linear potential a modulational instability can develop, in agreement with the Floquet theory analysis of Fig. 2. A good agreement was found also for other values of $\gamma$ and other initial conditions (at $\gamma=0$ the stability was checked only by numerical integrations of the original equation, since in this case $t=2 \pi / \gamma \rightarrow \infty$ and the Floquet theory becomes unapplicable). In particular we checked that the modulational instability of the background develops at later values of $t$, as $\gamma$ increases, and that an initial background with $k=\pi$ becomes again stable for $\gamma \sim 5.4$, in agreement with Fig.2.

\section{B. Dynamics of small amplitude pulses}

The fact that Eq. (28) possesses an integral of motion of type (29), implies that it cannot support solitary waves [23], i.e. it can not have solutions moving with constant velocity $v$ and depending on $n, t$, through the combination $n-v t$ (this is true also for $\gamma=0$ ). On the other hand, one can show that localized smooth excitations of small amplitude can propagate along the array with a relatively weak distortion. To investigate this dynamics, it is convenient to introduce a small parameter $\mu \ll 1$ defined as the square root of the ratio between the deviation from the background and the background itself, and consider the case of very small $\gamma$, i.e. we take $\gamma=o\left(\mu^{3}\right)$. This allow us to look for solutions of the form

$$
q_{n}=\left[\rho+\mu^{2} a\right] e^{i[\chi+\mu \phi]},
$$

where $a=a_{0}+\mu^{2} a_{1}+O\left(\mu^{4}\right)$ and $\phi=\phi_{0}+\mu^{2} \phi_{1}+O\left(\mu^{4}\right)$ are real functions of the slow variables $\xi=\mu n, T=\mu t$, $\tau=\mu^{3} t$, considered to be continuous and independent. We can then perform a multiple scale expansion 21 of Eq. (35), substituting $\gamma t$ with $\delta \tau$, with $\delta \sim o(1)$. A straightforward algebra shows that the expansion equation at the first order in $\mu$ is identically satisfied by the substitution (41).

FIG. 5. Time dependence of coefficients of Eq. (46) for $\delta=0.1, \rho=1$.

The equations at the orders $O\left(\mu^{2}\right)$ and $O\left(\mu^{3}\right)$ are

$$
a_{0}=-\frac{1}{4 \rho}\left[\partial_{T} \phi_{0}+2 \sin (\delta \tau) \partial_{\xi} \phi_{0}\right]
$$

and

$$
\begin{aligned}
& \partial_{T}^{2} \phi_{0}+4 \sin (\delta \tau) \partial_{T} \partial_{\xi} \phi_{0}+ \\
& 4\left[(\sin (\delta \tau))^{2}-\rho^{2} \cos (\delta \tau)\right] \partial_{\xi}^{2} \phi_{0}=0,
\end{aligned}
$$

respectively. It is worth to note that Eq. (43) at times $\delta \tau_{0}= \pm \pi / 2$ changes from hyperbolic (supporting wave propagation if $\cos (\delta \tau)>0)$ to elliptic. Let us consider the dynamics of the small amplitude excitation during the time in which Eq. (43) is of the hyperbolic type. We introduce a new running variable $\zeta=\xi-V(\tau) T$ where

$$
V(\tau)=2 \sin (\delta \tau)+2 \rho \sqrt{\cos (\delta \tau)}
$$

can be interpreted as slowly varying velocity of the wave packet (for the sake of definiteness we have chosen only one branch of the solution, the other branch being characterized by the "velocity" with opposite sign). One can then show that Eq. (43) is satisfied for arbitrary pair of functions $a_{0}(\zeta, \tau), \phi_{0}(\zeta, \tau)$ linked by

$$
a_{0}(\zeta, \tau)=\frac{1}{2} \sqrt{\cos (\delta \tau)} \partial_{\zeta} \phi_{0}(\zeta, \tau)
$$

In order to find the dependence of those functions on $\tau$, one has to consider the equations of the forth and fifth orders in $\mu$ (more precisely the condition of their compatibility). After tedious but straightforward calculations, one arrives at the following Korteweg - de Vries (KdV) equation

$$
\partial_{\tau} a_{0}+b_{1}(\tau) a_{0} \partial_{\zeta} a_{0}+b_{2}(\tau) \partial_{\zeta}^{3} a_{0}+b_{0}(\tau) a_{0}=0
$$

with slowly (if $\delta=o(1)$ ) varying coefficients $b_{j}(\tau)$ given by

$$
\begin{aligned}
& b_{0}(\tau)=\frac{1}{4} \delta \tan (\delta \tau), \quad b_{1}(\tau)=2 \frac{3[\cos (\delta \tau)]^{3 / 2}-\rho \sin (\delta \tau)}{\cos (\delta \tau)} \\
& b_{2}(\tau)=\frac{1}{12 \rho}\left(4 \rho \sin (\delta \tau)+\rho^{2}[\cos (\delta \tau)]^{1 / 2}-3[\cos (\delta \tau)]^{3 / 2}\right)
\end{aligned}
$$

FIG. 6. Evolution of the localized excitation in a BEC array, governed by Eq. (46). The initial wave profile corresponds to the pure $\mathrm{KdV}$ soliton (Eq. (47)) with parameters $u_{0}=1, \beta=1$. 
In Fig.6 we report the time evolution of a numerical solution of Eq. (46) with initial condition

$$
a_{0}(\zeta, \tau)=3 v \operatorname{sech}^{2}\left(\frac{\zeta-v \tau}{l_{c}}\right), \quad l_{c}=\left(4 \frac{\beta}{v}\right)^{1 / 2}
$$

Two important features can be seen from this figure. The first is the existence of a breaking time $\tau_{b r}$ below which the profile of the wave is distorted but its evolution is smooth (in Fig. 6 $\tau_{b r} \sim 0.5$ ). At $t=\tau_{b r}$ the breaking of the wavefront occurs, after which a train of solitary pulses (each of them being a $\mathrm{KdV}$ soliton) is emitted. This is similar to the generation of shock waves in a fluid (nonlinear Schrödinger lattice shock waves were first studied in Ref. 21]). Note that at the breaking time the coefficient $b_{2}\left(\tau_{b r}\right)$ is exactly zero (see Fig. 5), and Eq. (46) reduces to a known shock dynamics equation 38]. An essential difference between the case at hand and the case $\gamma=0$ treated in Ref. 21], however, is that in this last shock waves can appear only for a given carrier wave background, while here the linear potential (gravitational field of BEC arrays) makes them possible to exist for any wavenumber. Note that for the observation of shock waves it is important that $\delta$ is small compared with the amplitude of the background, this ensuring the time at which the group velocity dispersion becomes negligible, be long enough for shock waves to develop.

The second important feature which emerges from Fig. 6 is the possibility to have bright pulses propagating on top of a nonzero background (i.e. on top of a constant atomic population along the array). These pulses actually are ES, similar to those predicted in [20] for $\gamma=0$. For $\gamma \neq 0$, these excitations can undergo Bloch oscillations. To show this we have performed numerical integrations of Eq. 35), with a sech initial profile of large amplitude (i.e. comparable with the background level). The results are reported in Fig.7 (the numerical scheme used is the same of the previous section). We note that for the same parameter values, the bright excitation (i.e. above background) breaks down while the dark one (below background) remains stable for long times.

FIG. 7. Bloch oscillations of bright (a) and dark (b) localized excitations. Initial conditions:

$q_{n}(0)=\rho \pm 2 \eta \operatorname{Sech}[2 \eta n] \exp \left[i \phi_{n}(0)\right], \rho=0.1, \quad \eta=0.04$, $\gamma=0.1, \quad \phi_{n}(0)=-\frac{\pi}{2} n$.

An important conclusion following from these numerical studies is that bright and dark solitons of KdV type on top of homogeneous backgrounds, can exist in BEC array with positive scattering lengths.

\section{ARRAY OF BEC WITH NEGATIVE SCATTERING LENGTHS}

A distinctive feature of BECs with attractive interactions is the possibility of collapse when the number of atoms in the condensate exceeds a critical value $N_{c}$. From GPE one can predict $N_{c} \sim 1400$ for ${ }^{7} \mathrm{Li}$ atoms, a value which was confirmed experimentally in Ref. [39]. Arrays of optical traps can be used for manipulation of BECs with negative scattering length, if their wells are loaded with number of atoms less than this critical value. In this situation it is reasonable to consider the dynamics of ES on zero background, as described by Eq. (13) with $\sigma=1$. Bloch oscillations of bright ES were numerically investigated in Ref. [24]. From these simulations, a strong dependence of the dynamics on the amplitude of the initial wave was found (large amplitude wavepackets are quickly reduced to fragments, while small amplitude excitations keep their integrity over many oscillation periods). From numerical studies is also known that the amplitude of a dynamically localized ES can oscillate in time. Using the method of the previous section, we can develop analytical considerations on Bloch oscillations in BEC arrays with negative scattering lengths which explain the origin of these oscillations. We consider the case of small amplitudes and small $\gamma$, i.e. we assume, as before, $\mu=\sqrt{\gamma}$ to be a small parameter of the problem. Introducing the variable

$$
q_{n}=\psi_{n} \exp (-i \gamma n t)
$$

Eq. (13) can be written as

$$
\begin{gathered}
i \dot{q}_{n}+\cos (\gamma t)\left(q_{n+1}+q_{n-1}-2 q_{n}\right)+ \\
i \sin (\gamma t)\left(q_{n+1}-q_{n-1}\right)+2\left|q_{n}\right|^{2} q_{n}=0
\end{gathered}
$$

whose solution can be searched of the form

$$
q_{n}=\mu Q(\xi, \tau)
$$

with $\xi(\tau)=\mu n-x(\tau), \tau=\mu^{2} t$, and

$$
x=\frac{2}{\mu}(1-\cos \tau) \text {. }
$$

Note that $x(\tau)=O\left(\mu^{3}\right)=O\left(\gamma^{3 / 2}\right)$ and thus the substitution is self-consistent (at $\mu^{3} t \ll 1$ the term $\cos (\tau)$ can be expanded in Taylor series). After calculations analogues to those of the previous section, one arrives at the following nonlinear Schrödinger (NLS) equation with periodically varying dispersion

$$
i \partial_{\tau} Q+\cos (\tau) \partial_{\xi}^{2} Q+2|Q|^{2} Q=0
$$

The dynamics of this equation was numerically investigated starting with initial conditions of the form

$$
Q(\xi, 0)=2 \eta \operatorname{sech}\left(2 \eta\left(\xi-\xi_{0}\right)\right) .
$$

For the numerical code we used a split-step fast Fourier transform technique [40] with a time step $\Delta \tau=0.001$ and a space step $\Delta \xi=0.02$ (in normalized units), corresponding to 1024 grid points in the discretization domain taken as $-10 \div 10$ (absorbing boundary conditions were used to simulate an infinite domain). The accuracy of 
the scheme was checked by monitoring the conservation of the number of atoms and of the Hamiltonian, which was within $\pm 0.1 \%$ for all runs. The results are depicted in Fig.8.

FIG. 8. (a) Evolution of the localized excitation governed by Eq. (51), for $\eta=0.5$. and (b) Decay of its amplitude.

From Fig. 8a) we see that while the ES is executing spatial oscillations, its amplitude is oscillating in time. In Fig. 8b) we report the time dependence of the center of the wave on a longer time scale, from which we see that the amplitude while oscillating is also decaying (note, however, that this dynamics reproduces the dynamics of a real array only for times $\tau$ such that $\Delta \tau<1$ ). A more detailed numerical investigation shows that the amplitude can be either decaying or growing in time, depending on initial conditions (the growth can reach also the $60 \%$ of the initial amplitude of the pulse). This implies that if at $t=0$ the number of atoms in one well is below the critical value $N_{0}(t=0)<N_{c r}$ for collapse, the growth in amplitude due to the linear potential, can induce collapse after a time $t=t_{c r}$ at which $N_{0}\left(t_{c r}\right)=N_{c}$.

\section{CONCLUSIONS}

The dynamical properties of BEC arrays with positive and negative scattering lengths have been studied in the framework of a discrete nonlinear Schrödinger equation derived from the mean field GPE with a tight-binding approximation. The interplay between macroscopic inter site tunnelling and nonlinear self-trapping was shown to be responsible for the appearance of different types of DMS. In particular we showed, both analytically and numerically, that for a small ratio between the tunnel coupling constant and the nonlinear interatomic interactions, ILM solutions can exist. For BEC arrays with non-zero backgrounds the modulational stability problem was investigated and the existence of bright and dark ES was discussed. The problem of Bloch oscillations of envelope solitons in arrays with positive scattering lengths was also analytically and numerically investigated. We showed that at the lower orders of a perturbative expansion, the dynamics of small amplitude excitation evolves according to a $\mathrm{KdV}$ equation with time dependent coefficients. In this case the possibility of shock waves formation, in BEC arrays with positive scattering lengths, was explicitly displayed. In the case of arrays with negative scattering lengths, the dynamics of small amplitude excitations was described in terms of a nonlinear Schrödinger equation with a periodically varying dispersion. This equation was used to show the presence of amplitude oscillations during Bloch oscillations, as well as decay or growth of the excitations, depending on the initial conditions. The results of this paper clearly show the complexity and the wide range of behaviors which can arise in the system.
In closing this paper we feel compelled to discuss to which extend the phenomena presented in this paper could be observable in real BEC arrays. Our model is based on a tight binding approximation, this putting restrictions on the shape of the wavefunctions, as well as, on the potential profile, to be used. Although a detailed analysis of the experimental settings for which the tight binding approximation is accurate has not yet been done, there are experimental situations in which this is obviously true. Thus, for example, if the potential barrier is wide and tall enough to reduce the tunnelling probability among adjacent BECs, the overlapping of the wavefunctions is certainly small and the model is accurate. In this case DMS of the type discussed above should appear.

In particular, the possibility to observe ILMs in real experiments should not be overlooked. We remark that from Eqs. (7), (12), it follows that the peak amplitude, i.e. $\max \left(\phi_{n}(t)\right)$, is of order $O(1)$, so that the atomic population can be localized on very few sites of the array. In the experiment in Ref. [11], the peak density was $n_{0}=$ $10^{13} / \mathrm{cm}^{3}$, the mean field energy $g_{0} n_{0} \approx k_{B} \cdot 4 \mathrm{nK}\left(k_{B}\right.$ being the Boltzmann constant), this giving the parameter $\kappa$ in Eq. (18) to be $\kappa \approx 1$. To observe ILM one needs to have at least $\kappa \sim 0.1$. This can be achieved either by increasing the number of atoms in each well (from $10^{3}$ to $10^{4}$ ), or by reducing the tunnelling constant $K$, which exponentially depends on the lattice constant (distance between potential wells). When the number of atoms in the wells is increased, however, a loss of coherence of the condensate can occur, a phenomenon observed in the experiments reported in Ref. [32]. On the other hand, $\kappa$ can be reduced also by changing the atomic scattering length $a_{s}$ using the Feshbach resonances [33], so that it should be possible to find experimental settings for which ILM can be observed.

As to $\mathrm{ES}$ on finite backgrounds discussed in Section IV, we remark that the number of atoms involved in these $\mathrm{KdV}$ solitons can be estimated as $N_{s}=$ $2 \mu \rho \int_{-\infty}^{\infty} a\left(\zeta / l_{c}\right) d \zeta=4 \mu u_{0} \rho l_{c}$. For the parameters used in the numerical simulations of Fig. 7, with $\delta=\mu^{3}=$ $0.1, u_{0}=\beta=1$ we have $\mu \approx 0.46, l_{c}=3.464$, and for an array of Rb atoms with 1000 atoms in each well, we have $N_{s} \sim 6370$ atoms. These bright (dark) solitons on top of a background, could be created by using a laser beam applied for short time to a uniform BEC array, so to create a local enhancement (depletion) of the potential. In this case the dependence of the soliton velocity on its amplitude is an interesting parameter to measure since, in contrast with nonlinear Schrödinger solitons, the velocity of $\mathrm{KdV}$ solitons depends on the amplitude of the wave (number of the atoms in the condensate). This could provide a way to experimentally check our results.

We hope that experiments in this direction will be soon performed. 


\section{ACKNOWLEDGMENTS}

F.Kh.A., B.B.B. and S.A.D. are grateful to the US CRDF (Award ZM2-2095) for partial financial support. V.V.K. acknowledges support from FEDER and Program PRAXIS XXI, grant $\mathrm{N}^{o}$ Praxis/P/Fis/10279/1998 and the EC grant HPRN-CT-2000-00158. F.Kh.A. and V.V.K. acknowledge NATO fellowship program for the support of cooperative work. M.S. thanks the "Centro de Física da Matéria Condensada", University of Lisbon, for a one month Visiting Professorship, the EC grant HPRNCT-1999-00163, and the MURST-PRIN-2000 Initiative, for financial support.

$\dagger \quad$ Electronic address: fatkh@physic.uzsci.net

$\ddagger \quad$ Electronic address: baizakov@physic.uzsci.net

2 Electronic address: sdarmanyan@yahoo.com

$3 \quad$ Electronic address: konotop@cii.fc.ul.pt

$4 \quad$ Electronic address: salerno@sa.infn.it

[1] A. Smerzi, S. Fantoni, S. Giovanazzi, and S.R. Shenoy, Phys. Rev. Lett. 79, 4950 (1997).

[2] G.J. Milburn, J. Corney, E.M. Wright, and D.F. Walls, Phys.Rev. A 55, 4318 (1997).

[3] I. Zapata, F. Sols, and A. Leggett, Phys.Rev., 57, R23, 1998.

[4] K. Likharev, Theory of Josephson Junctions, (Nauka, Moscow, 1988).

[5] J.E. Williams, Optimal conditions for observing Josephson oscillations in a double-well Bose-gas condensate, eprint:arXiv:cond-mat/0102148.

[6] Sun Zhang, Fan Wang, Phys. Lett. A279,231 (2001).

[7] For a review see F. Dalfovo et al., Rev. Mod. Phys. 71, 463 (1999); J. Javanainen, Phys.Rev. A 60, 4902 (1999).

[8] A. Trombettoni and A. Smerzi, Phys. Rev. Lett. 86, 2353 (2001).

[9] A.C. Scott Nonlinear Science: Emergence and dynamics of coherent structures, Oxford University Press, (1999).

[10] M.R. Andrews, C.G. Townsend, H.-J. Miesner, D.S. Durfee, D.M. Kurn, and W. Ketterle, Science, 275(0), 637, (1997).

[11] B.P. Anderson, M.A. Kasevich, Science, 282, 1686 (1998).

[12] Q. Niu, X. Zhao, G.A. Georgakis, and M.G. Raizen, Phys. Rev. Lett., 76, 4504 (1996); D.I. Choi, Q. Niu, Phys. Rev. Lett., 82, 2022 (1999); M.B. Dahan, E. Peik, J. Reichel, Y. Castin, and C. Salomon, Phys. Rev. Lett., 76, 4508 (1996); K.Berg-Sorensen, K. Molmer, Phys. Rev. A58, 1480 (1998).

[13] In some literature the word discrete breather appears as synonymous of ILM. Since breathers solutions close to the continuum (i.e. localized on many lattice sites) can also exist in these systems and thus can be interpreted as envelope solitons, we prefer not to introduce this terminology.
[14] One can estimate from Fig. 4 of Ref. 8] a width of their excitations of about 30 lattice sites.

[15] B. Denardo, B. Galvin, A. Greenfield, A. Larraza, S. Putterman, and W.Wright, Phys.Rev.Lett. 68, 1730 (1992); P. Marquie, J. M. Bilbaut, and M. Remoissenet, Phys.Rev.E 51, 6127 (1995); H. S. Eisenberg, Y. Silberberg, R. Morandotti, A. R. Boyd, and J. S. Aitchison, Phys. Rev. Lett. 81, 3383 (1998).

[16] A.J.Sievers and S.Takeno, Phys. Rev. Lett. 61, 970 (1988); J. B. Page, Phys. Rev. B 41, 7835 (1990).

[17] S. Darmanyan, A. Kobyakov, and F. Lederer, Sov. Phys. JETP 86, 682 (1998); S. Darmanyan, A. Kobyakov, F. Lederer, and L. Vázquez, Phys. Rev. B 59, 5994 (1999).

[18] Y.S.Kivshar, W. Krolikowski, and O. A. Chubykalo, Phys. Rev. E 50, 5020 (1994); V. Konotop and S. Takeno, Phys. Rev. E 54, 2010 (1996).

[19] S. Flach and C.R.Willis, Phys. Rep. 295, 181 (1998); F. Lederer, S. Darmanyan, A. Kobyakov, in: Optical Solitons, edited by W. Torruellas and S. Trillo, (Springer,Heidelberg, 2001) (in press).

[20] O.A. Chubykalo, V. V. Konotop, and L. Vázquez, Phys. Rev. B 47, 7971 (1993).

[21] V. V. Konotop and M. Salerno, Phys. Rev. E 55, 4706 (1997); ibid 56, 3611 (1997).

[22] V. V. Konotop and S. Takeno, Phys. Rev. B 60, 1001 (1999).

[23] M. Salerno, B. A. Malomed, and V. V. Konotop, Phys. Rev E 62, 8651 (2000).

[24] R. Scharf and A. Bishop, Phys.Rev. A 43, 6535 (1991).

[25] M. Bruschi, D. Levi, and O. Ragnisco, Nuovo Cimento A 53, 21 (1979); D. Cai, A. R. Bishop, N. Grønbech-Jensen, and M. Salerno, Phys. Rev. Lett. 74, 1186 (1995). V. V. Konotop, D. Cai, M. Salerno, A. R. Bishop, and N. Gronbeh-Jensen, Phys. Rev. E 53, 6476 (1996).

[26] C. Kittel, Introduction to the Solid State Theory,(Cambridge UP, 1975).

[27] W.P. Reinkhardt and C.W. Clark, J.Phys. B 30, L785 (1997).

[28] J. Denschlag, J.E. Simsarian,, D.L. Feder et al., Science 287, 97 (2000); S.Burger, K. Bongs, S. Dettmer et al. Phys.Rev.Lett. 83, 5198 (1999).

[29] S.Potting, P.Meystre, and E.M. Wright, Atomic solitons in optical lattices, e-print con-mat/00009289v2, (2000).

[30] E. Hairer, S.P. Norsett and G. Wanner, Solving Ordinary Differential Equations I. Nonstiff Problems. 2nd edition. Springer series in computational mathematics, (SpringerVerlag,Heidelberg, 1993).

[31] E. W. Laedke, O. Kluth, and K. H. Spatschek, Phys. Rev. E 54, 4299 (1996); M. Johansson and S. Aubry, Nonlinearity 10, 1151 (1997).

[32] M.O. Mewes et al., Phys. Rev. Lett. 78, 562 (1997)

[33] S. Inouye, M.R. Andrews, J. Stenger, H.-J Miesner, D.M. Stamper-Kurn, and W. Ketterle, Nature 382,151 (1998).

[34] V. V. Konotop, O. A. Chubykalo, and L. Vázquez, Phys. Rev. E 48, 563 (1993);

[35] V. V. Konotop, Teor. Mat. Fiz. 99, 413 (1994);

[36] A.H. Nayfeh, Introduction to Perturbation Techniques, (Wiley, N.Y., 1981).

[37] W.H. Press, S.A. Teukolsky, W.T. Vetterling, and B.P. Flennary, Numerical Recipes. The Art of Scientific Com- 
puting. (Cambridge Univ. Press, 1996).

[38] G.B. Whitham, Linear and nonlinear waves, (Wiley, N.Y., 1974).

[39] C.C. Bradley, C.A. Sackett, and R.G. Hulet, Phys. Rev. Lett., 78, 985 (1997).

[40] G.P. Agrawal, Nonlinear Fiber Optics. 2nd edition. (Academic Press, 1995). 


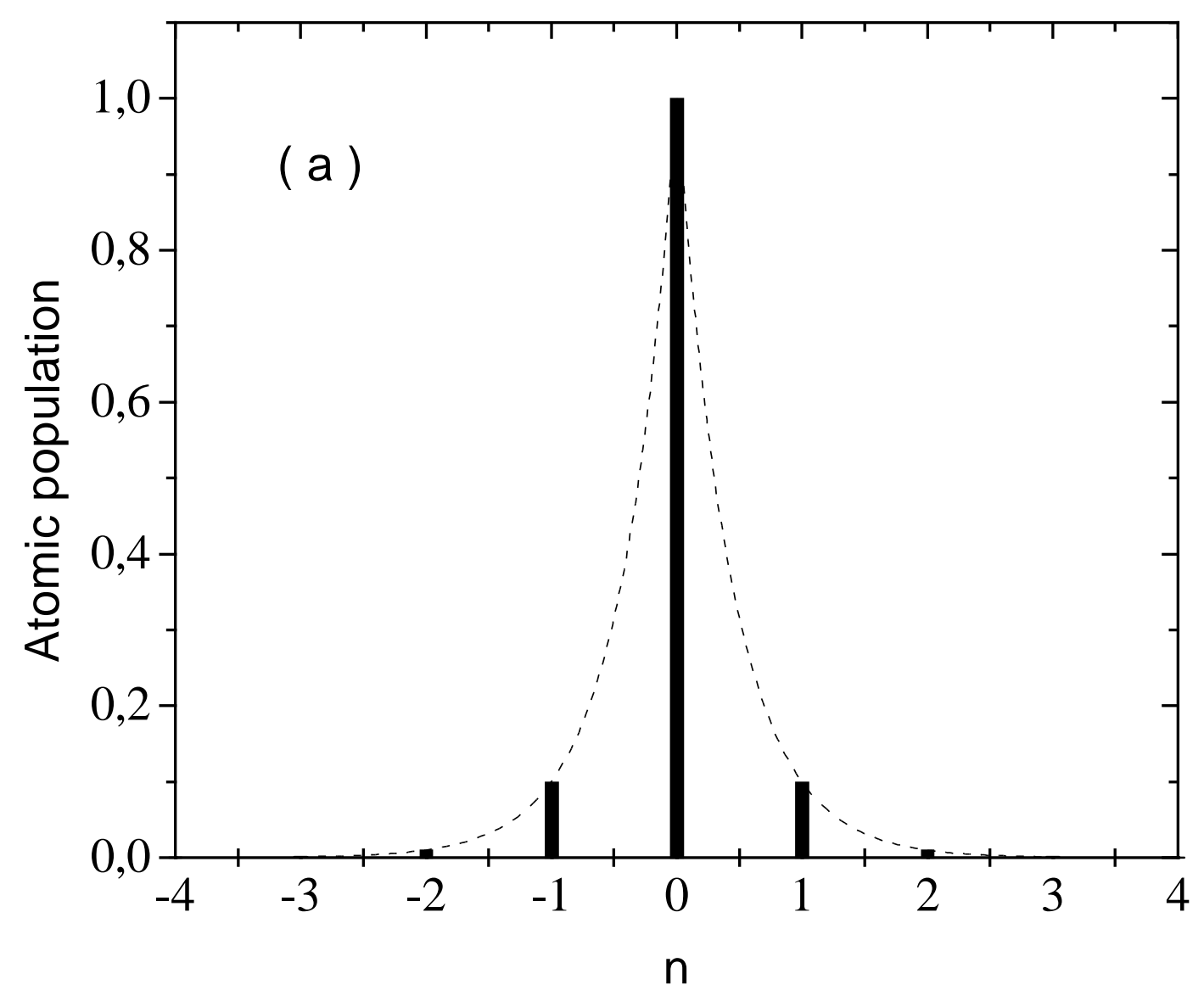




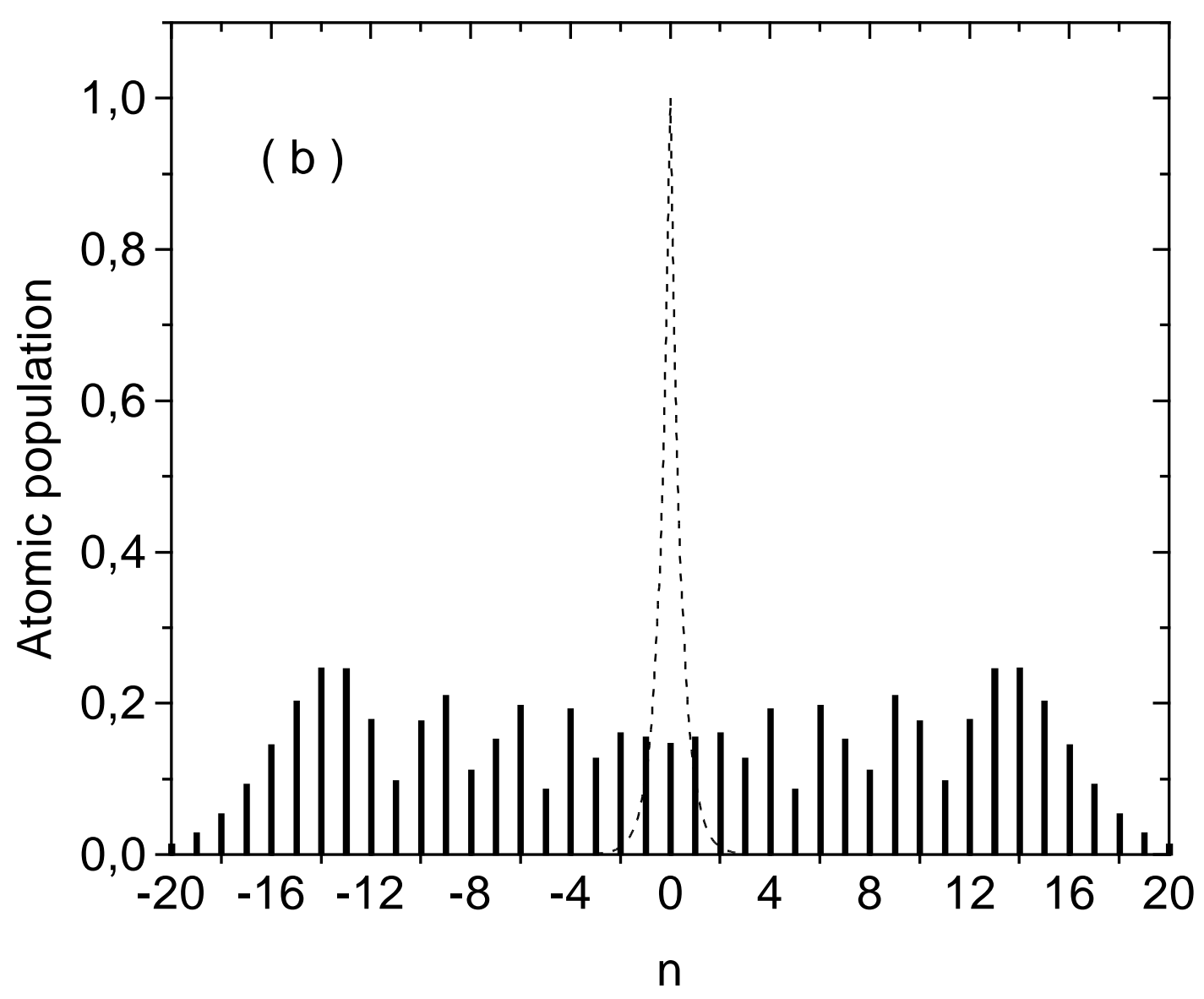




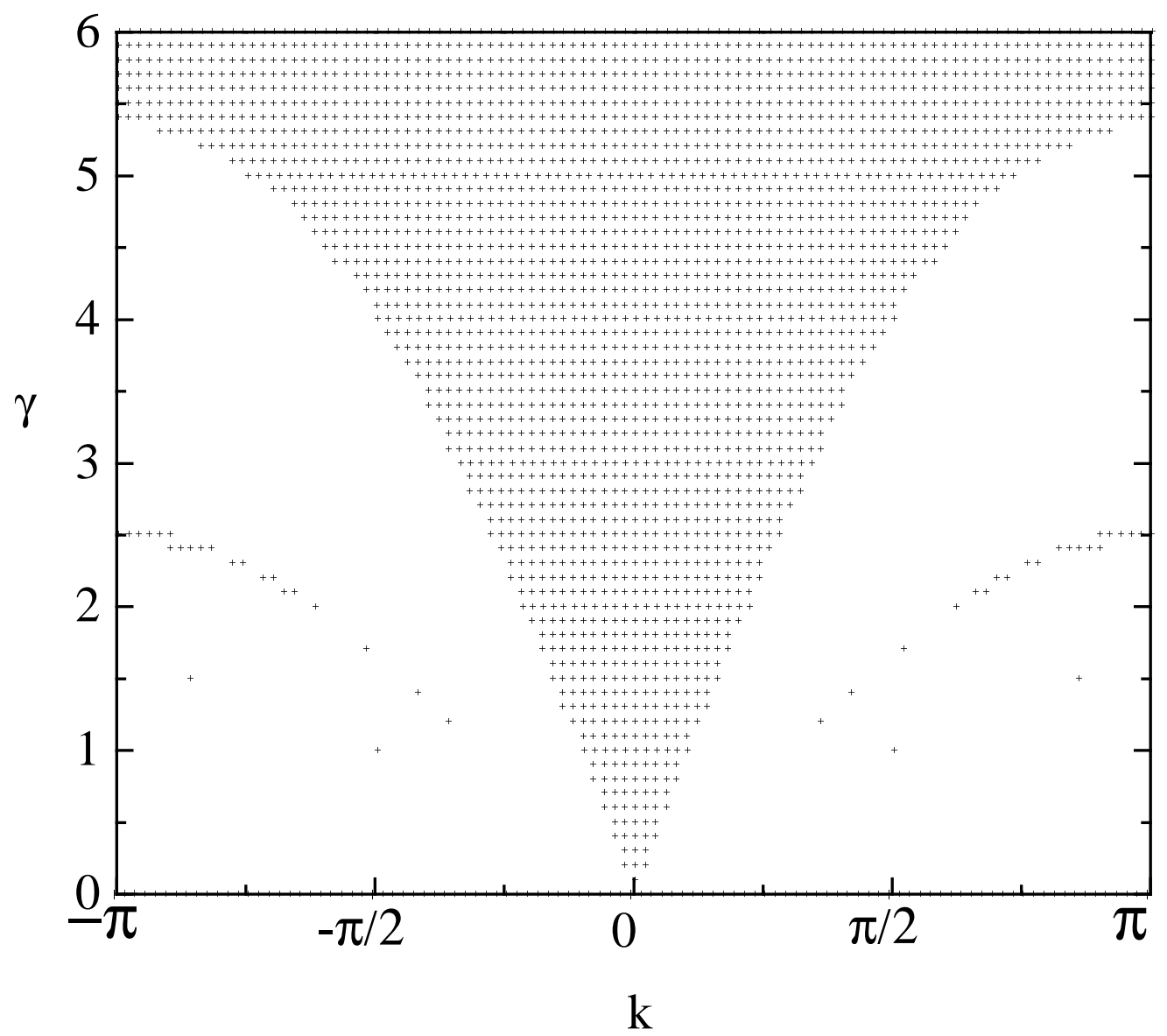




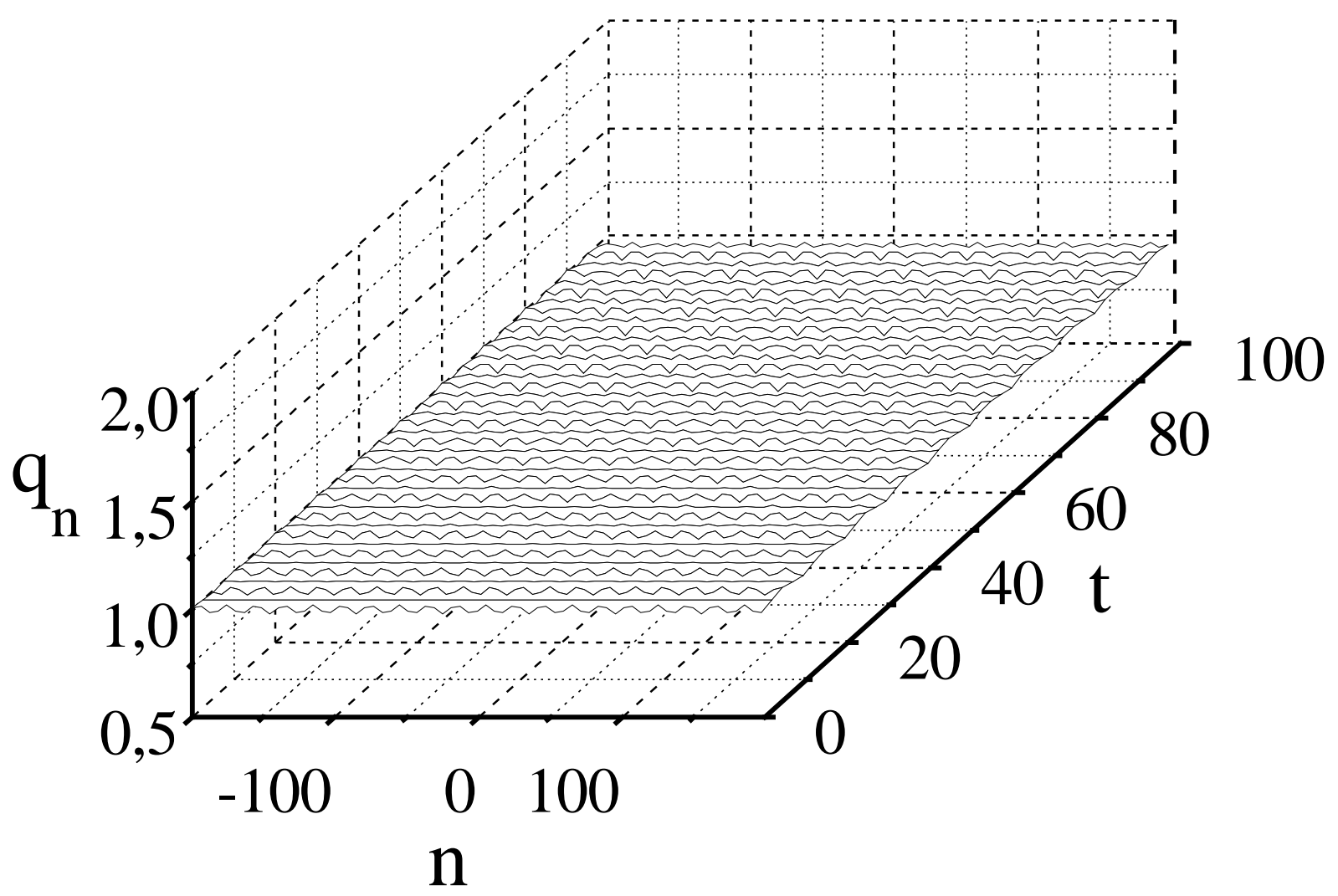




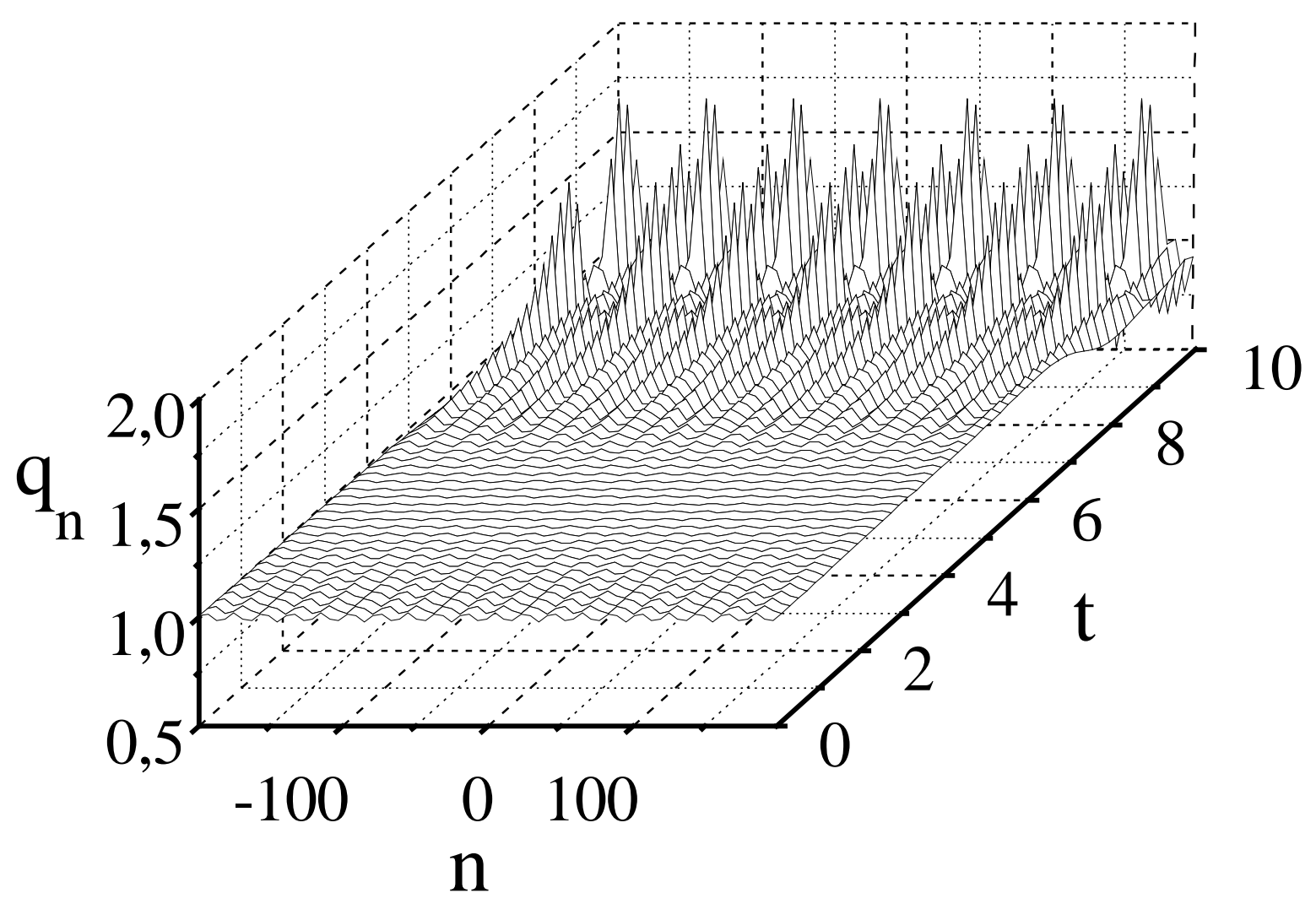



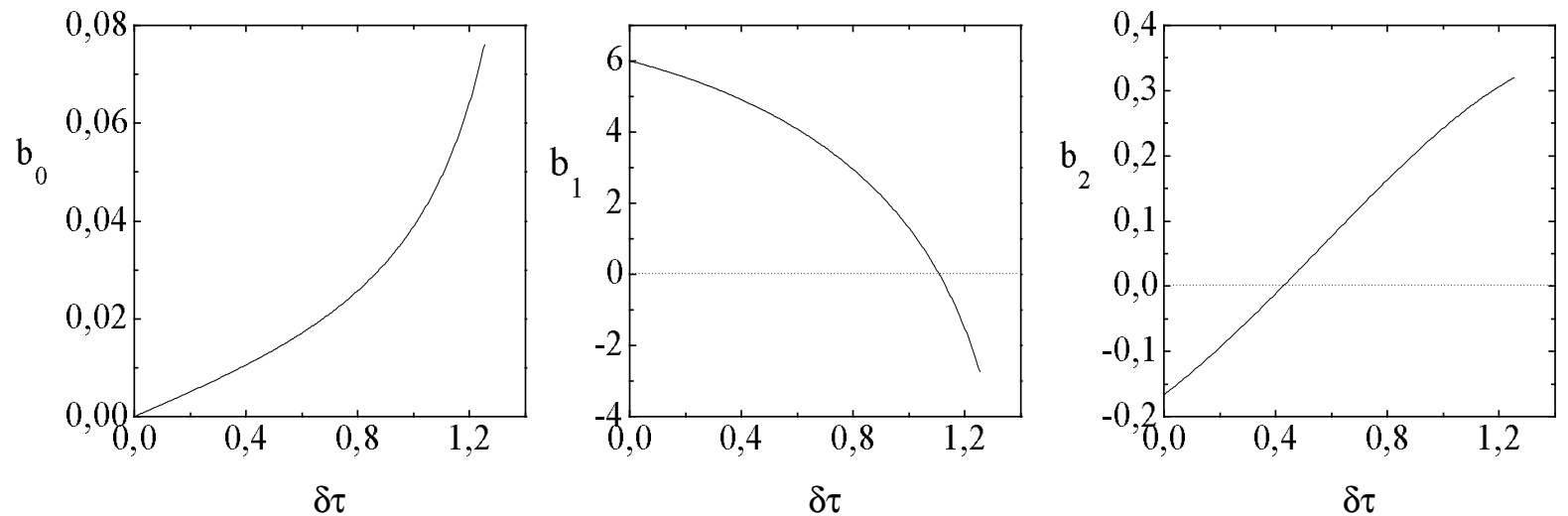


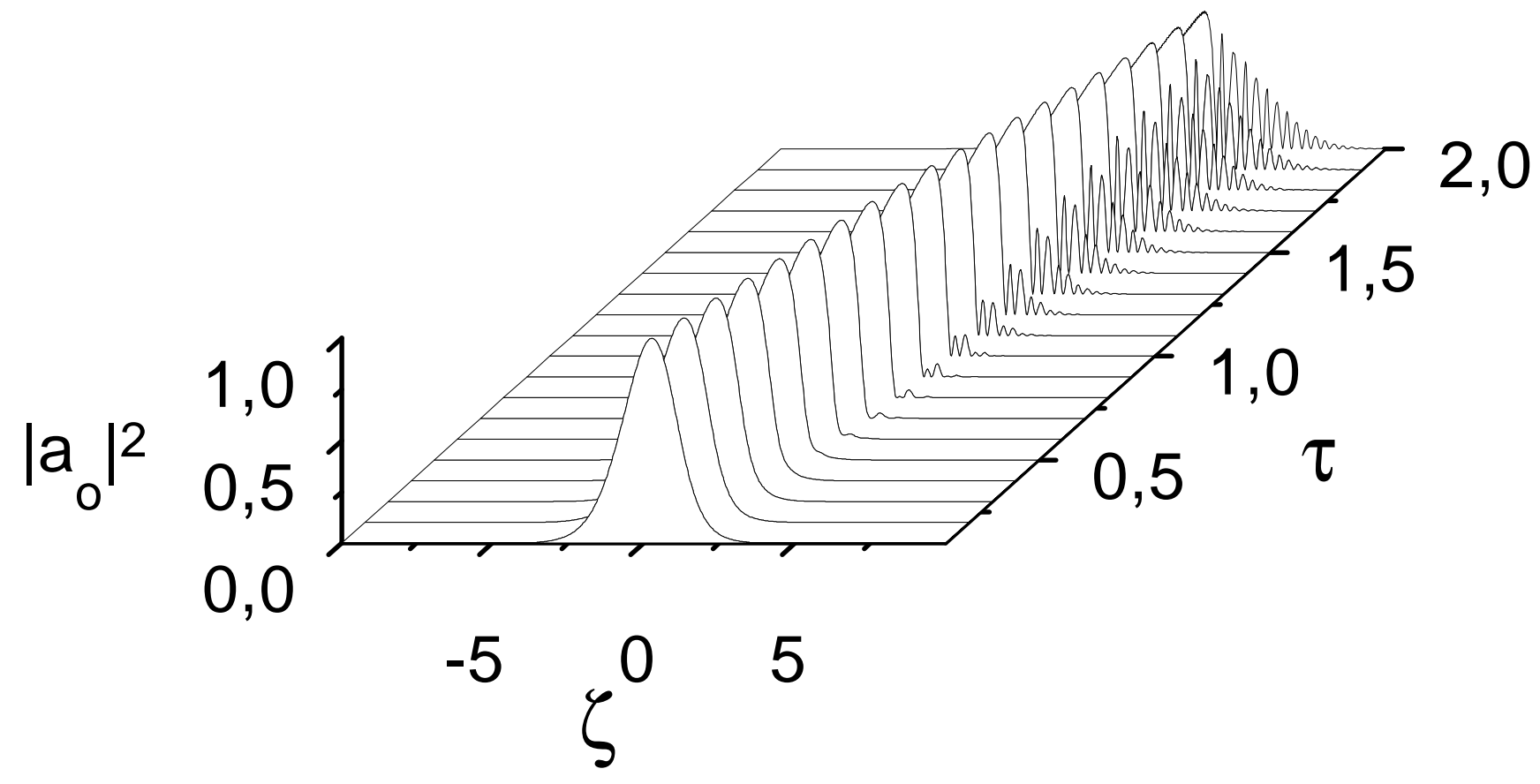




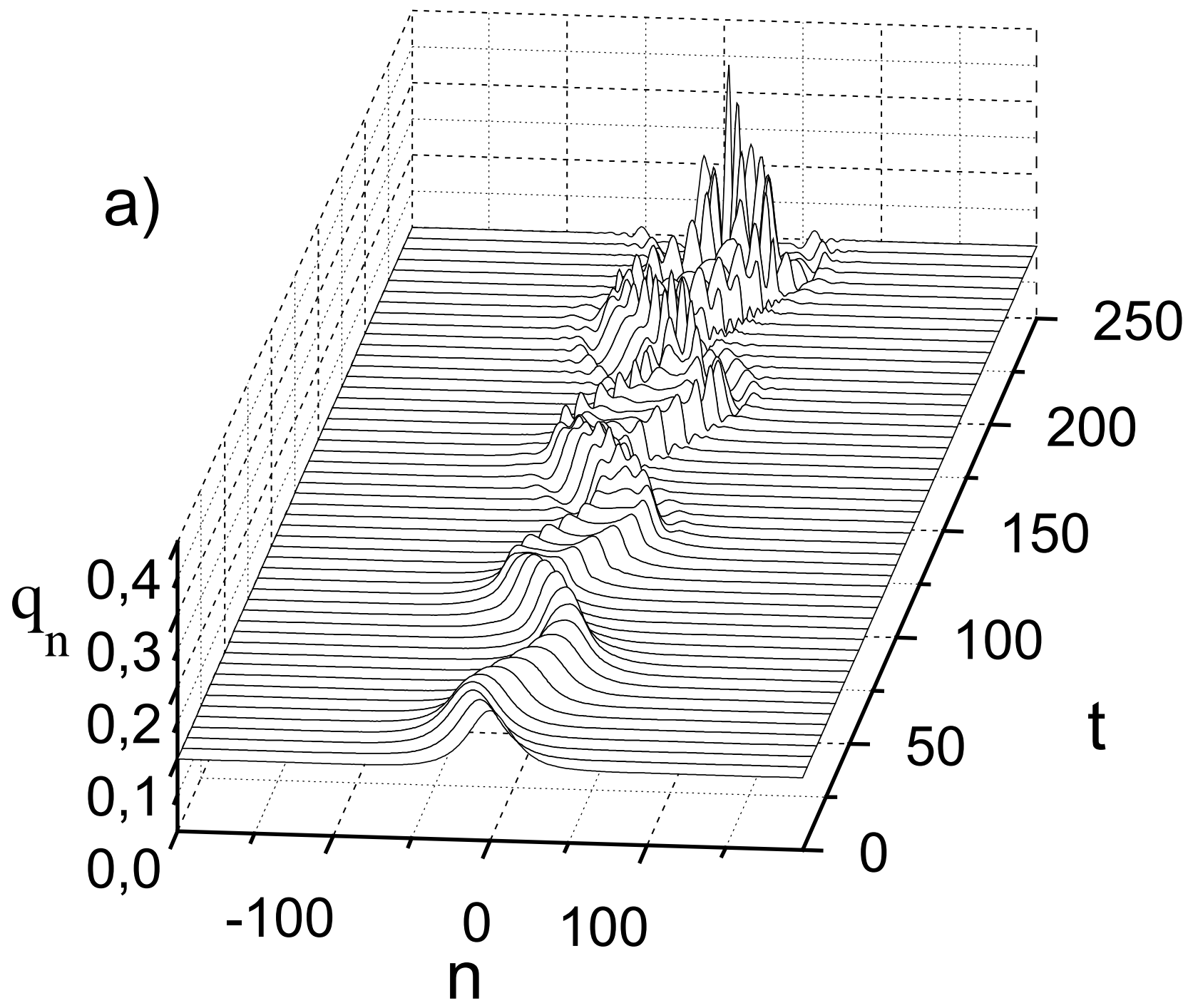




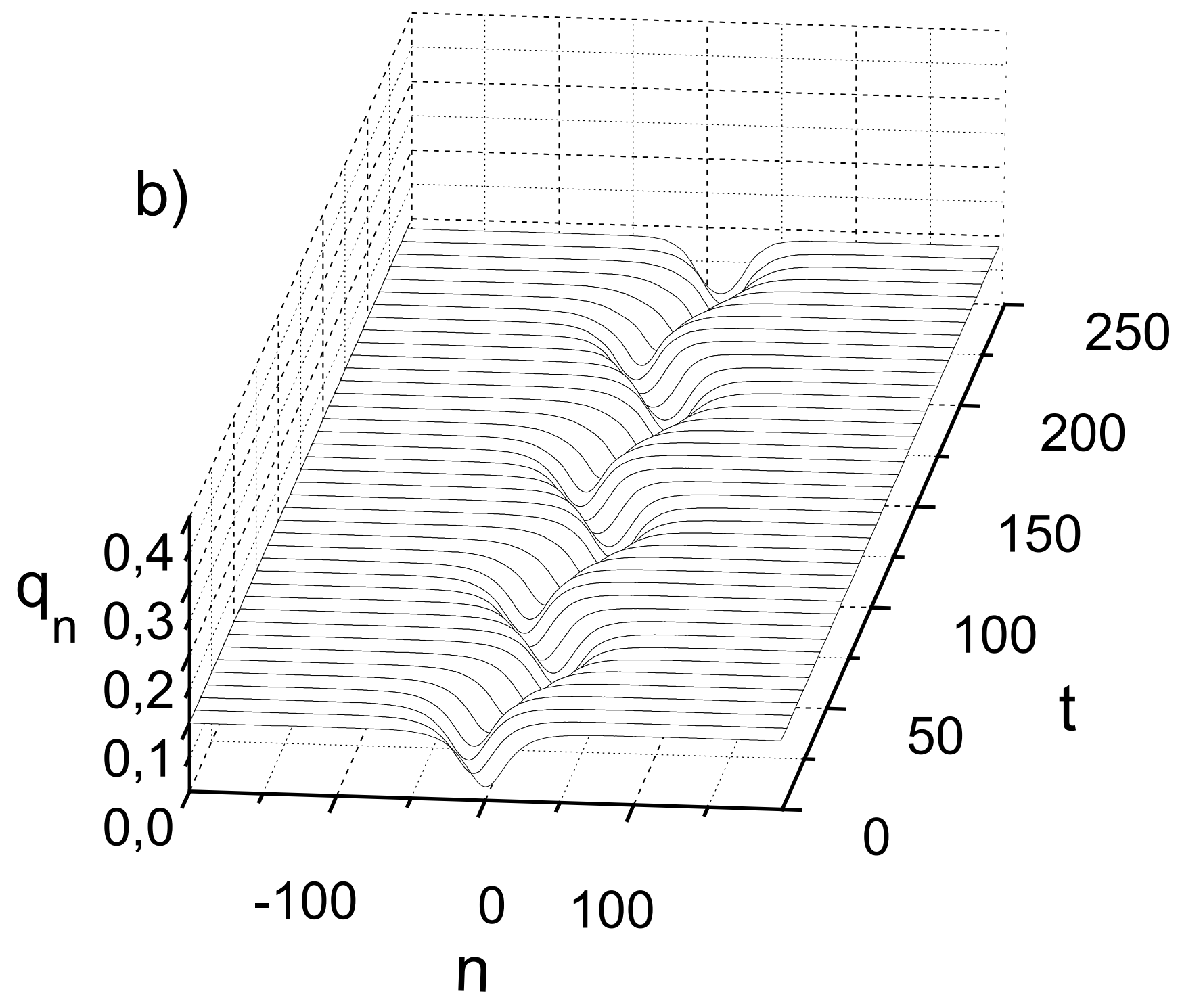




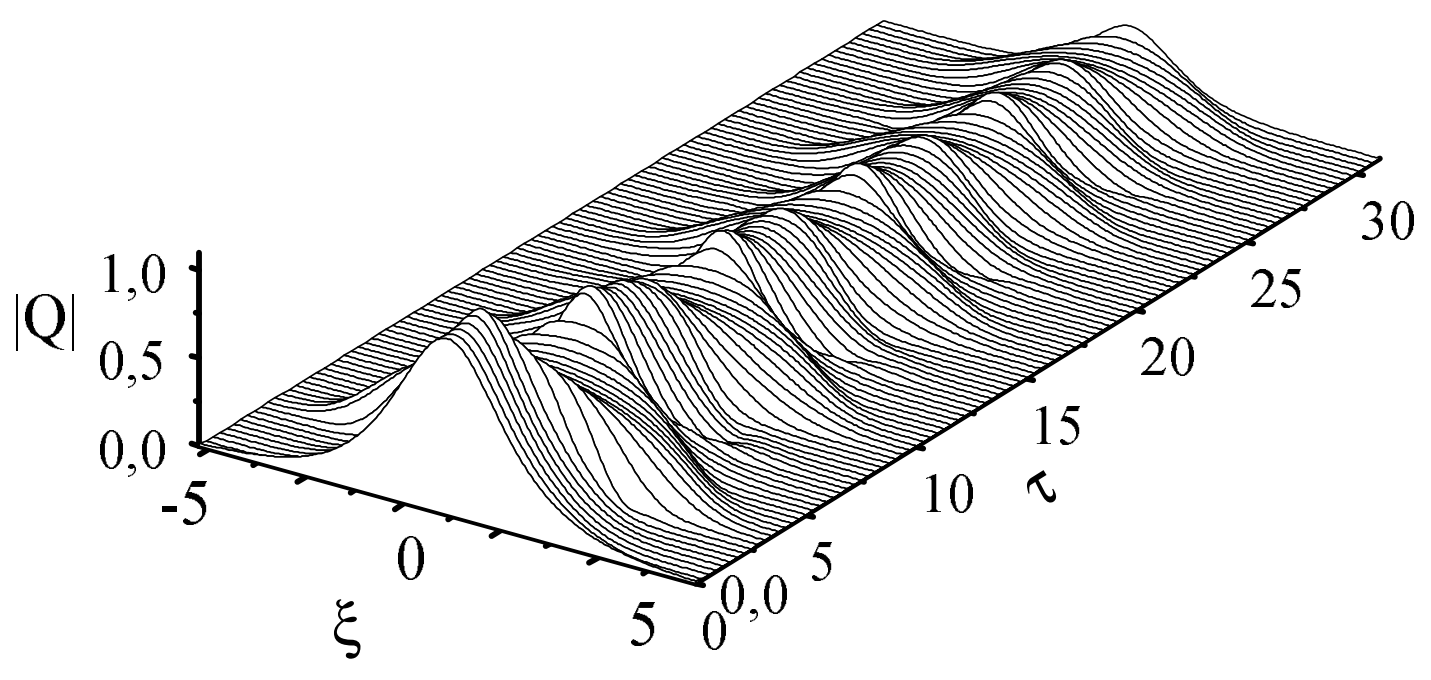




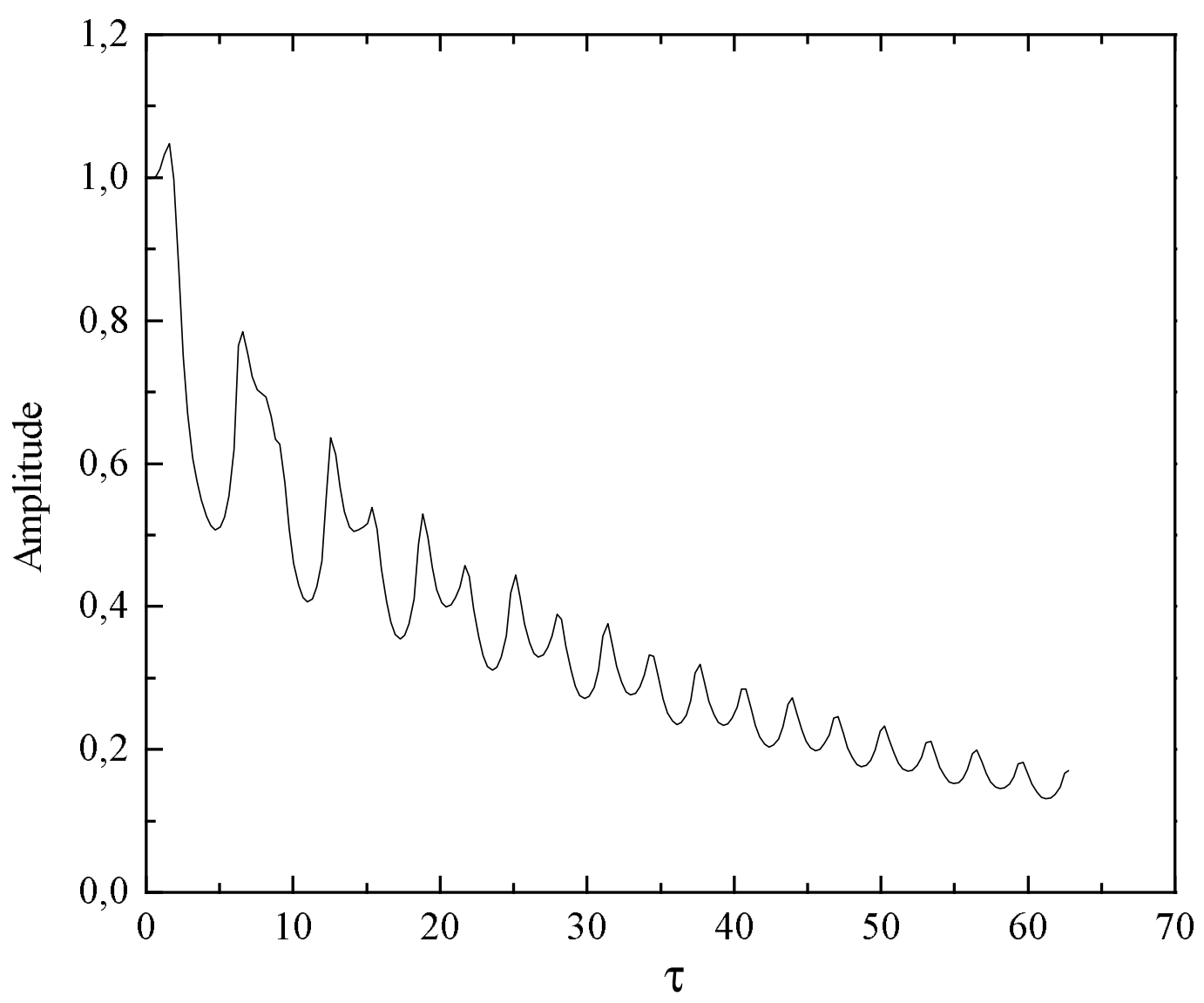

